\title{
Das Curriculum für Hausarztmedizin am USZ - eine Erfolgsgeschichte
}

\author{
Die 2-jährige Weiterbildung zur Erlangung hausarztspezifischer Kompetenzen am \\ UniversitätsSpital Zürich ist derart beliebt, dass sogar Bewerber abgelehnt werden \\ müssen. Eine Ausweitung des Angebotes zur Sicherstellung des Nachwuchses in der \\ Grundversorgung wäre sinnvoll.
}

Thomas Rosemann, Elisabeth Bandi-Ott, Ryan Tandjung

1 Haller U, Tschudi P, Bally K, Isler R. Berufsziel von Medizinstudierenden. Primary Care. 2005;5(20):468-72.

2 Badertscher N, Schöni M, Berger L, Zoller M, Rosemann T. Weiterbildung von angehenden Hausärzten an stationären Weiterbildungsstellen: Sicht der Assistenzärzte. Praxis. 2011;100(6):349-55

Korrespondenz: Prof. Dr. med. Thomas Rosemann, PhD Direktor Institut für Hausarztmedizin und Versorgungsforschung der Universität Zürich Pestalozzistrasse 24 CH-8091 Zürich Tel. 0442559855 Fax 0442559097 thomas.rosemann[at]usz.ch
Wie in den meisten europäischen Nachbarländern besteht auch in der Schweiz ein zunehmender Hausärztemangel, insbesondere in ländlichen Regionen. Alarmierend sind Daten aus einer Erhebung von Haller, Tschudi et al. [1] unter Studierenden, die zeigen, dass sich nur noch etwa 10\% für den Beruf des Hausarztes interessieren.

Eine Studie des Institutes für Hausarztmedizin, die unter Assistenzärzten in Spitälern durchgeführt wurde, kam zu einem etwas anderen Bild: Hier äusserten immerhin $45 \%$ der Befragten, dass sie sich durchaus vorstellen könnten, Hausärztin oder Hausarzt zu werden [2]. Da der Nachwuchsmangel mittlerweile aber auch die Spezialgebiete in den Spitälern erfasst hat, sieht sich dieser Nachwuchs heute stark umworben, und da ist es natürlich naheliegend, eine «pfannenfertige» Weiterbildung etwa in der Kardiologie oder Nephrologie oder einer anderen Spezialität einzugehen. Die Hausarztmedizin kann diese Angebote in der Regel nicht offerieren, insbesondere eine breite Weiterbildung, die auch die «kleinen», aber für die spätere Tätigkeit immens wertvollen Fächer, wie etwa die Dermatologie, ORL oder Rheumatologie, umfasst, ist für den angehenden Hausarzt innert nützlicher Zeit nicht realisierbar. Weiterbildungsstellen in den genannten Fachgebieten sind oft schwer zu bekommen, wenn man nicht den entsprechenden Facharzttitel oder einen geringeren Zeitraum als ein Jahr anstrebt. Damit würde eine breitgefächerte Facharztausbildung jedoch weit länger dauern als die vorgesehenen 5 Jahre, von dem logistischen Aufwand für den angehenden Hausarzt einmal ganz abgesehen.

In Verhandlungen mit der Gesundheitsdirektion des Kantons Zürich wurde am UniversitätsSpital daher auf den 1.1.2010 ein Curriculum für Hausarztmedizin institutionalisiert. Die Gesundheitsdirektion stellt dafür pro Jahr etwa 550000 Franken zur Verfügung. Mit diesen Mitteln können prinzipiell etwa 4-5 Assistenzarztstellen geschaffen werden. In Verhandlungen mit den Klinikdirektoren der ORL, Dermatologie und Rheumatologie wurde erreicht, dass die entsprechenden Kliniken spezielle Weiterbildungsstellen für ein Hausarztcurriculum zur Ver-

\section{Le succès du cursus de formation} en médecine de famille à l'USZ \section{ne se dément pas}

La Suisse s'attend à une pénurie de médecins de famille. Une mesure pour y pallier consiste à proposer aux futurs généralistes une formation structurée leur permettant d'acquérir des compétences spécifiques. L'article ci-après présente le cursus de formation sur deux ans proposé conjointement par l'Institut de médecine de famille et l'Université de Zurich et qui bénéficie du soutien du canton de Zurich. Proposé en sus de la formation postgraduée de base pour le titre de spécialiste en médecine interne générale, ce cursus prévoit 3 rotations de 6 mois en $\mathrm{ORL}$, en dermatologie et en rhumatologie, ainsi qu'un assistanat de 6 mois en cabinet médical. Le succès de ce cursus de formation est tel qu'il faut malheureusement refuser des candidats faute de moyens. Cela prouve qu'une formation attrayante et structurée répond à un réel besoin. II serait donc judicieux d'étendre l'offre afin d'assurer la relève en médecine de premier recours.

fügung stellen. Zudem beteiligen sie sich zu $50 \%$ an den Personalkosten, was zu einer Verdopplung der Stellen führt. Die Praxisassistenz wird zu 25\% vom Lehrpraktiker mitfinanziert.

Das Curriculum richtet sich an künftige Grundversorgerinnen und Grundversorger, die ihre internistische Basisweiterbildung bereits absolviert haben. Das zweijährige Curriculum sieht insgesamt drei 6-monatige Rotationen in den Fächern Oto-RhinoLaryngologie, Dermatologie und Rheumatologie vor, ergänzt mit einer 6-monatigen Praxisassistenz. Die 
Weiterbildung in den Spezialfächern erfolgt in den Weiterbildungsstätten des UniversitätsSpitals Zürich, die Praxisassistenz ist innerhalb des Kantons Zürich frei wählbar. Für die Weiterbildungsphase in den spezialisierten Fachgebieten wurden vor dem Programmstart in Zusammenarbeit mit den Kliniken spezifische Lernziele formuliert. Zusätzlich zur Organisation der Stellen sind eine Begleitung durch eine Mentorin und der Besuch von regelmässigen Fortbildungsveranstaltungen am Institut für Hausarztmedizin vorgesehen.

\section{Das Curriculum richtet sich an künftige Grundversorger, die ihre internistische Basisweiterbildung bereits absolviert haben.}

Neben den oben erwähnten klinischen Aufnahmebedingungen für das Programm, sieht der Kanton Zürich innerhalb 5 Jahren nach Abschluss des Curriculums eine Niederlassungspflicht als Grundversorgerin oder Grundversorger im Kanton vor.

Vier Kollegen haben das Programm - bzw. Teile davon - bereits abgeschlossen, drei weitere werden das Curriculum noch dieses Jahr abschliessen. Von den bisherigen vier Absolventen sind bereits zwei im Kanton Zürich in der Grundversorgung tätig, eine dritte Kollegin steht vor dem Praxisstart im Verlauf dieses Herbstes. Die Nachfrage nach dem Curriculum sprengt alle Erwartungen und passt so gar nicht zum Bild der oft kolportierten «unattraktiven» Hausarztmedizin. Vielmehr bestätigt es die Ergebnisse unserer Studie, die zeigt, dass viele Ärzte in Weiterbildung für die Hausarztmedizin zu gewinnen wären, wenn

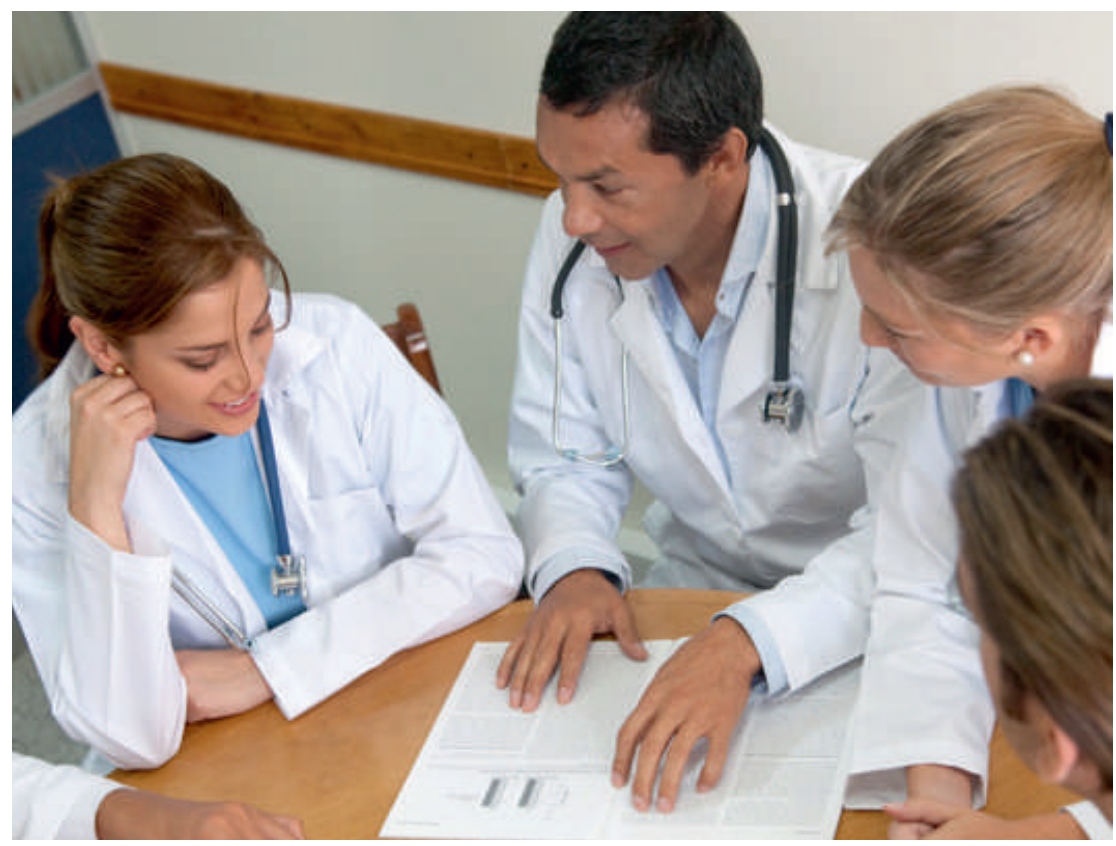

Mentoring der jungen Hausärzte in Weiterbildung ist ein wichtiger Bestandteil im Curriculum. man ihnen adäquate und zu den Spezialdisziplinen konkurrenzfähige Weiterbildungsangebote machen würde. Wöchentlich erhalten wir Anfragen von interessierten Kolleginnen in der Weiterbildung, bis 2014 sind jedoch bereits alle Stellen besetzt, ohne dass das Programm aktiv beworben wird. Diese Wartezeiten führen leider dazu, dass vielen Bewerbern keine Stelle angeboten werden konnte. Seit Beginn des Programmes haben insgesamt 14 Personen entweder die Bewerbung meist aufgrund der Wartezeiten zurückgezogen oder konnten unsererseits nicht für eine Stelle berücksichtigt werden.

\section{Vor- und Nachteile unseres Programmes}

Das zweijährige Curriculum Hausarztmedizin bietet ein strukturiertes Programm für künftige Hausärztinnen und Hausärzte an. Die sechsmonatigen Rotationen in den drei erwähnten Fachgebieten erlauben es den Weiterzubildenden, sich zusätzliches Wissen und Fertigkeiten aus diesen Spezialgebieten anzueignen, die für ihre künftige Tätigkeit von unschätzbarem Nutzen sind. Die Teilfinanzierung erlaubt es den Kliniken, Weiterbildungsstätten auch für eine kürzere Phase künftigen Hausärztinnen und Hausärzten anzubieten; ebenso profitieren sie von internistisch gut vorgebildeten Ärztinnen und Ärzten.

Einschränkend für unser Programm sind die mit sieben Stellen eher knapp bemessenen Ressourcen, die dazu führen, dass viele Interessierte nicht berücksichtigt werden können. Zudem ist die fixe Rotation in den drei Fächern aktuell gegeben, womit insbesondere die Stellenplanung der involvierten Kliniken berücksichtigt wird; letzter Punkt führt auch dazu, dass Teilzeitstellen - obwohl der Bedarf sehr hoch ist - nicht angeboten werden können. Ausserdem ist es zurzeit nur möglich, das ganze Programm zu absolvieren, kürzere Phasen können nicht unterstützt werden.

Wünschenswert ist ein grösserer Stellenetat, der neben mehr Stellen vor allem eine zusätzliche Flexibilität für weitere Rotationen (z. B. Pädiatrie, Gynäkologie) zulässt, die nach Wünschen und Bedürfnissen der künftigen Grundversorgerinnen und Grundversorger angepasst werden könnten. Ein Etat explizit für Praxisassistenzen, wie kürzlich im Kanton Bern bewilligt, muss auch für den Kanton Zürich selbstverständlich sein. Die zusätzliche Hürde mit der Niederlassungspflicht innerhalb des Kantons Zürich (bzw. der Rückzahlungspflicht bei Nichteinhalten) ist störend; werden flächendeckend ähnliche Angebote auch in anderen Teilen der Schweiz angeboten, ist es vielleicht auch politisch möglich, von dieser Niederlassungspflicht wegzukommen.

\section{Fazit}

Insgesamt zeigt der überwältigende Erfolg des Curriculums am USZ, dass die Hausarztmedizin sehr wohl eine Option für Ärzte in Weiterbildung ist - man muss sie nur attraktiv anbieten. 